\title{
A New Correlation-Based Granulometry Algorithm with Application in Characterizing Porous Silicon Nanomaterials
}

\author{
Ricardo Hitoshi Maruta, Hae Yong Kim, \\ Danilo Roque Huanca and Walter Jaimes Salcedo
}

Escola Politécnica, Universidade de São Paulo, Av. Prof. Luciano Gualberto, tr. 3, 158, 05508-010, São Paulo, Brazil.

E-mails: rhmaruta@gmail.com, hae@lps.usp.br, droqueh@gmail.com,wsalcedo@1me.usp.br.

Granulometry is the process of measuring the size distribution of objects in an image of granular material. Usually, algorithms based on mathematical morphology or edge detection are used for this task. We propose a entirely new approach for the granulometry using the cross correlations with circles of different sizes. This technique is primarily adequate for detecting circular-shaped objects, but it can be extended to other shapes using other correlation kernels. Experiments show that the new algorithm is greatly robust to noise and can detect even faint objects. This paper also reports the quantitative structural characteristics of the porous silicon layer based on the proposed algorithm applied to Scanning Electron Microscopy (SEM) images. The new algorithm computes the size distribution of pores and classifies the pores in circular or square ones. We relate these quantitative results to the fabrication process and discuss the square porous silicon formation mechanism. The new algorithm shows to be reliable in SEM images processing and is a promising tool to control the pores formation process.

\section{Introduction}

Granulometry is the process of measuring sizes of different objects/grains in an image of granular material. The granulometric curve or pattern spectrum of an image is the histogram of objects as the function of radius. The objective of the granulometry is, given an image, to obtain its pattern spectrum. There are two main groups of image-based granulometry algorithms:

- Mathematical morphology-based algorithms;

- Edge detection-based algorithms.

Mathematical morphology-based granulometry obtains the pattern spectrum of an image without explicitly segmenting it. Dougherty et al. present a popular morphologybased granulometry for binary images (1). Raimundo et al. used this algorithm to characterize porous material (2). Unfortunately, this algorithm cannot be directly applied to grayscale images. If the original image is grayscale, the algorithm must somehow convert it into a binary image and any binarization discards many important information. Vincent presents a morphology-based granulometry for grayscale images (3). A demonstration program of this algorithm with source code is available at (4). This algorithm seems to be scarcely used in practice. Indeed, the output of this algorithm is highly non-intuitive, difficult to be used in practice. It represents the pattern spectrum as the "sum of pixel values in opened image as a function of radius." Ordinaly, the user wants to obtain simply the 
"quantity of objects as a function of radius." Moreover, in many applications the spatial localization of each grain is important, and this information is not provided by grayscale morphology granulometry. Edge detection-based granulometry detects the edges of the image using conventional gradient operators and thresholding (5). Then, it delimitates the objects using the edges. Edge-detection is a noise-sensible operation and may not be reliable, especially in blurred low-contrast images.

This paper presents a entirely new approach to the granulometry. We use cross correlations with circles of different sizes. This technique is primarily adequate for detecting circular-shaped grains, but it can detect directly square or equilateral triangle directly. It can also be extended to other shapes using non-circular correlation kernels. Experiments show that the new algorithm is greatly robust to noise and can detect even faint objects. The present work also reports the quantitative structural characteristics of the porous silicon layer based on the new algorithm applied to Scanning Electron Microscopy (SEM) images. These results allow us analyzing the square pores formation correlated to thermal annealing process of the silicon wafer that was metallized previously to the anodization process.

\section{Correlation-Based Granulometry}

The discrete cross correlation between two real-valued images $t$ and $a$ is defined as:

$$
t(x, y) \circ a(x, y)=\sum_{m} \sum_{n} t(m, n) a(x+m, y+n)
$$

In practice, images are defined only inside a rectangular domain. An image is considered to be filled with zeros outside of its domain. Cross correlation can be efficiently computed using FFT (Fast Fourier Transform). Cross correlation can be used to find a (usually small) template $t$ inside a (usually large) image $a$. This operation, called "template matching", computes the mean-corrected image $\tilde{t}=t-\bar{t}$ by subtracting the mean grayscale $\bar{t}$ from each pixel of image $t$; and then computes the cross correlation $c(x, y)=\tilde{t}(x, y) \circ a(x, y)$. The peaks of image $c$ corresponds to the occurrences of $t$ in $a$.

Cross correlation is an image contrast-dependent operation. That is, if many instances of $t$ with different contrasts occur in $a$, the instance with the largest contrast will correspond to the highest peak in $c$. For image-processing applications where the brightness/contrast of the images can vary due to illumination and exposure conditions, this is an undesirable property. So, to obtain brightness/contrast-invariance, the normalized cross correlation is usually applied instead of the cross correlation. However, for our application, the contrast-dependency allows us to distinguish clear objects (a high contrast instance in $a$ yields high peak in $c$ ) from faint objects (a low contrast instance in $a$ yields so low peak in $c$ ). Choosing an appropriate limiar to threshold image $c$ allow us to detect only the clear objects (high threshold) or even faint low-contrast objects (low threshold).

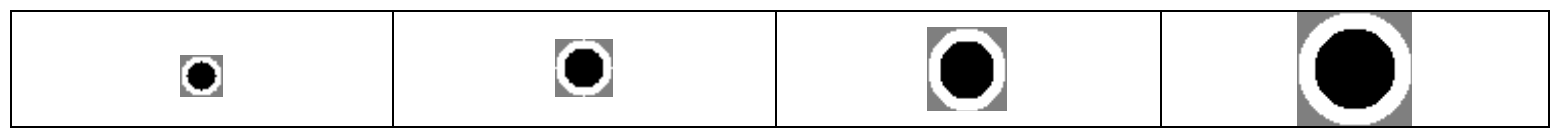

Figure 1. Some of the kernels used in our granulometry. Black pixels have negative values, white ones are positive, and gray ones are zeros.

To obtain the pattern spectrum of an image $a$ of granular material, we propose to compute cross-correlations of $a$ with circular kernels of different radii $t_{1}, t_{2}, \ldots, t_{n}$ (figure 
1). The sizes of the kernels increase in geometric progression. We have noticed experimentally that 5 kernels per octave are enough to obtain scale-invariance. The radii of inner and outer circles are chosen so that the number of black pixels (that correspond to negative values) are nearly equal to the number of white ones (that correspond to positive values). Gray pixels are zeros. In each kernel the sum of all negative pixels is -0.5 and the sum of all positive pixels is +0.5 . So, if the grayscale of the image ranges (for example) from 0 to 255, the image resulting from the correlation will range from -255 to 255 . Let us denote the images resulting from the cross correlation as $c_{1}, c_{2}, \ldots, c_{n}$, where:

$$
c_{i}(x, y)=\tilde{t}_{i}(x, y) \circ a(x, y)
$$

Let us define the maximal correlation image as:

$$
C(x, y)=\stackrel{n}{\operatorname{MAX}}\left[c_{i}(x, y)\right]
$$

A peak in $C(x, y)$ correspond to a (quasi) circular object in $a$. The scale of the detected object is given by the argument of the maximal correlation:

$$
G(x, y)=\underset{i=1}{\operatorname{ARMAX}}\left[c_{i}(x, y)\right]
$$

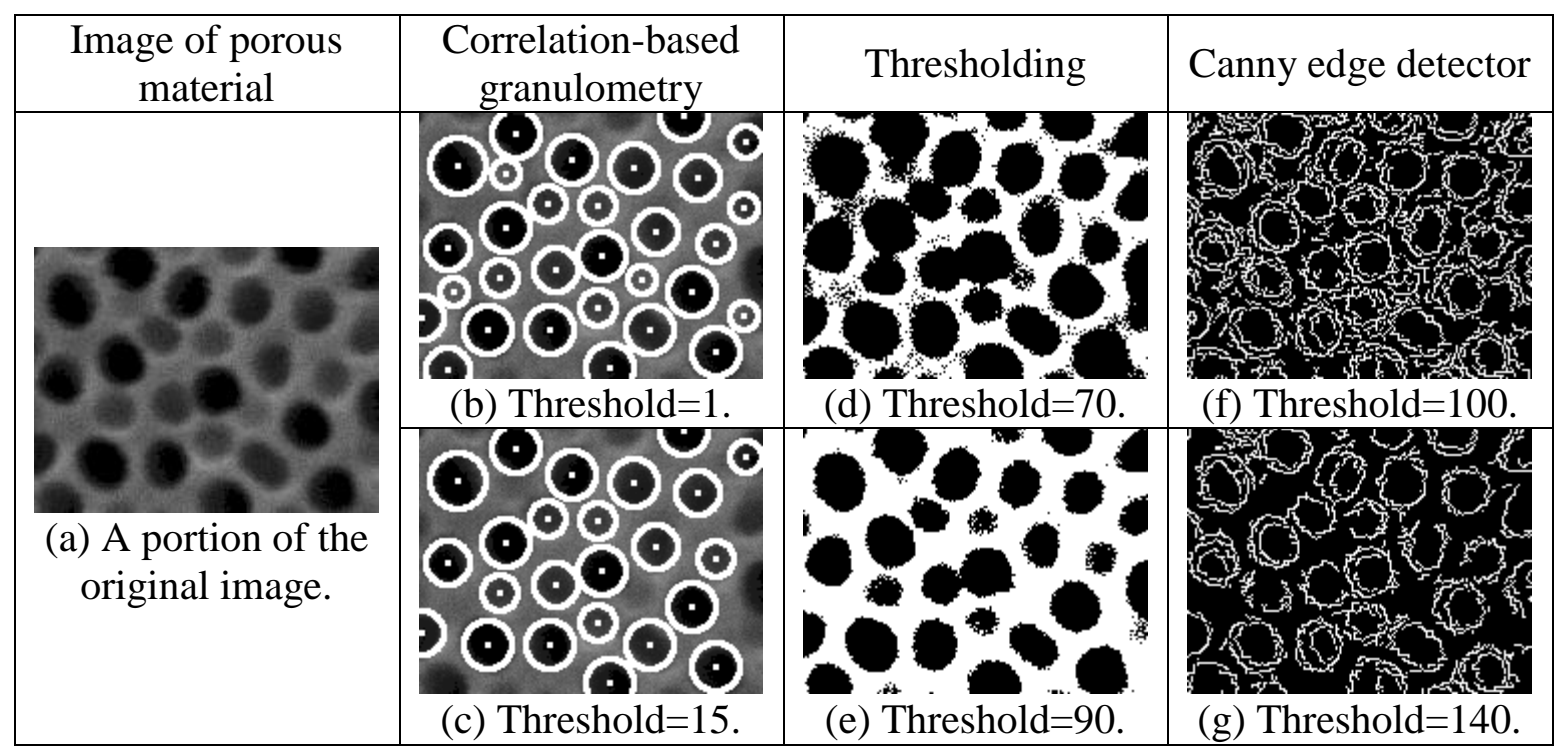

Figure 2. (a) A sub-image of a porous material image with 21 pores located entirely within the image (18 clear pores and 3 faint pores). (b-c) The output of our algorithm detecting all 21 pores or only 18 clear pores depending on the threshold limiar. (d-e) Thresholding with different parameters used as the first step of the morphology-based granulometry. (f-g) Canny edge detector used as the first step of the edge detection-base granulometry.

Our algorithm sorts the correlation peaks in decreasing order. A peak (that represents a circular object) is discarded if it intersects with some other peak with larger correlation in more than $S \%$ of the circular object's area, where $S$ is a user-defined threshold. This allows us detecting partially intersecting grains.

Figure 2 depicts the outputs of our algorithm and compare it with the outputs of thresholding (the preliminary step of binary morphology-based granulometry) and Canny 
edge detection (the preliminary step of edge-based granulometry). In figure $2 \mathrm{a}$, there are 21 pores that do not intersect with the image canvas, where 18 of them are clearly visible and 3 are faintly visible. Our algorithm successfully detected all the 21 pores or only the 18 clearly visible ones, depending on the chosen limiar. It is not possible to detect all the pores after the thresholding (figures $2 \mathrm{~d}$ and $2 \mathrm{e}$ ), because if the faint pores become visible (threshold $=70$ ) then the clear pores get merged. Similarly, it is not possible to detect after edge detection (figures $2 \mathrm{f}$ and $2 \mathrm{~g}$ ) because edge detector fails to clearly delimit all the pores. Moreover, the algorithm yielded double edges, due to the characteristics of the SEM images.

\section{Experiment}

The macroporous silicon layers with circular and square pores were obtained by electrochemical anodization process of the (100) p-type silicon wafer with resistivity of c.a. $10 \Omega \mathrm{cm}(6)$. The anodization process was carried out in the single electrochemical cell using HF(48\%): DMF mixture where HF correspond to $12 \%$ of total volume of the solution. The cell was anodically polarized at galvanostatic condition fixing the current density at $12 \mathrm{~mA} / \mathrm{cm}^{2}$ for $20 \mathrm{~min}$. The square pores layer was obtained from Al metallized silicon wafer. In order to control the square pores density the metallized wafers were annealed in the $\mathrm{N}_{2}$ environment for $1,2,4$ and 8 hours. The density of square pores at porous layer is of fundamental importance for silicon macrotubes formation. So, the present work reports the SEM images processing in order to control the experimental parameter for adequate macroporous silicon layer formation. The structural analysis was carried out by Scanning Electron Microscopy (SEM) techniques, specifically using NanoSem 400 microscopy. The images were obtained by excitation with electron beam of $10 \mathrm{kV}$ to 30 $\mathrm{kV}$ using the electron secondary technique. Table I shows the annealing times of the metallized silicon wafers and the thichkness of their porous layers formed after the anodization process.

TABLE I. Annealing time of metallized silicon wafer and thickness of porous layer formed after anodization process.

\begin{tabular}{|c|c|c|c|c|}
\hline Annealing time (hours) & 1.0 & 2.0 & 4.0 & 8.0 \\
\hline Thickness of MPS $(\mu \mathrm{m})$ & 24 & 34 & 32 & 38 \\
\hline
\end{tabular}

\section{Image Processing Results}

We wrote a C++ program named Granul specifically to analyze images of macroporous silicon layers with circular and square pores (figure 3a). This program consists of four sub-programs:

1. Corrcirc: Implements the proposed correlation-based granulometry to detect both circular and square pores. Figure $3 \mathrm{~b}$ depicts part of the output of this sub-program obtained by processing figure 3 a.

2. Classify: Classifies automatically the detected pores in circle or square (figure 3c), based on the size of the pore (blue circle) and on the "influence zone", that is, the pore's neighbor area where there is no other pore (yellow circle).

3. Edit: Allows to rectify manually eventual errors made by the automatic detection and classification (figure 3d). The manual correction is marked in green.

4. Relat: Computes the area of square pores by a seed growing algorithm (in red, figure $3 \mathrm{e}$ ) and generates the pattern spectrum. 


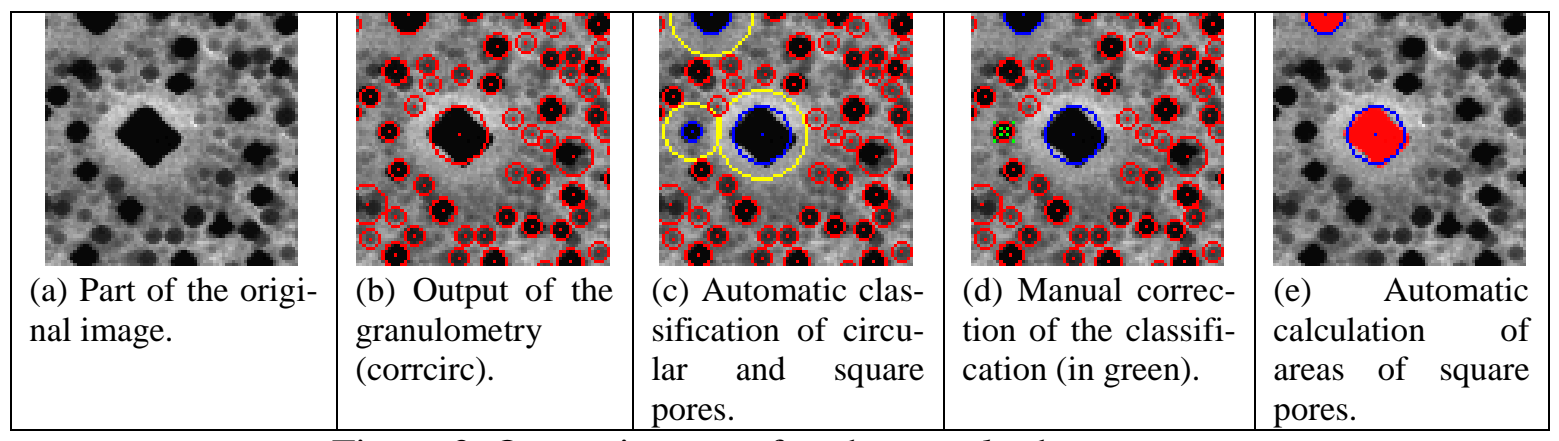

Figure 3. Output images of each granul subprograms.

The SEM images of macroporous silicon layers were annealed for different times are shown in figures $4 \mathrm{a}$ to $4 \mathrm{~d}$. These images show that the distributions of square and circular pores depend on the thermal annealing process. We applied the proposed algorithm to obtain the quantitative data of the pores structural features.

Figure 5a depicts the histogram of circular pores. The circular pores distributions are similar for all samples and the predominant pores are those with areas $0.48 \mu \mathrm{m}^{2}$. Figure $5 \mathrm{~b}$ shows the histograms of square pores. The samples annealed for one and two hours present low square pores density with similar distribution of areas ranging from 0.48 to $12.86 \mu \mathrm{m}^{2}$. The sample annealed for 4 hours presents the highest square pores density and the predominant square pores have areas of $3.72 \mu \mathrm{m}^{2}$. The sample annealed for 8 hours shows a significant decreasing of the square pores density and predominant pores have areas of $9.38 \mu^{2}$. These quantitative results give us important information to understand the mechanism of square pores formation. The square pores are important for the silicon macrotubes formation.

The annealing time of $\mathrm{Al}$ metallized sample before anodization process promoted the increasing on the sheet resistance (figure 6) and this behavior is well correlated with increasing of the square pores density observed in samples annealed for 1 to 4 hours. However, in the sample annealed for 8 hours, in spite of the sheet resistance increasing, the square pores density decreased. These results suggest that the sheet resistance value on silicon surface define the initial pitch distribution of pores formation during anodization process and, since the pitch formation is dependent on the electric field intensity, the field distribution on the silicon surface at initial stage of anodization process depends on the level of the sheet resistance and so the initial pitchs are originated from the breakdownlike process. In this sense, there is a critical field correlated with annealing time of sample such that the square pores density decreases. Since this critical field is high intensity, the area of pores can increase as consequency. Additionally, in the last case, the long annealing time could have induced Al metal clustering preferentially at some region where the initial pores pitch should be formed preferentially.

The SEM images analysis by the proposed granulometric algorithm yielded a quantitative information of the pores size and their distribution in circular and square pores. These results were important to understand the mechanism of square macropores formation. 


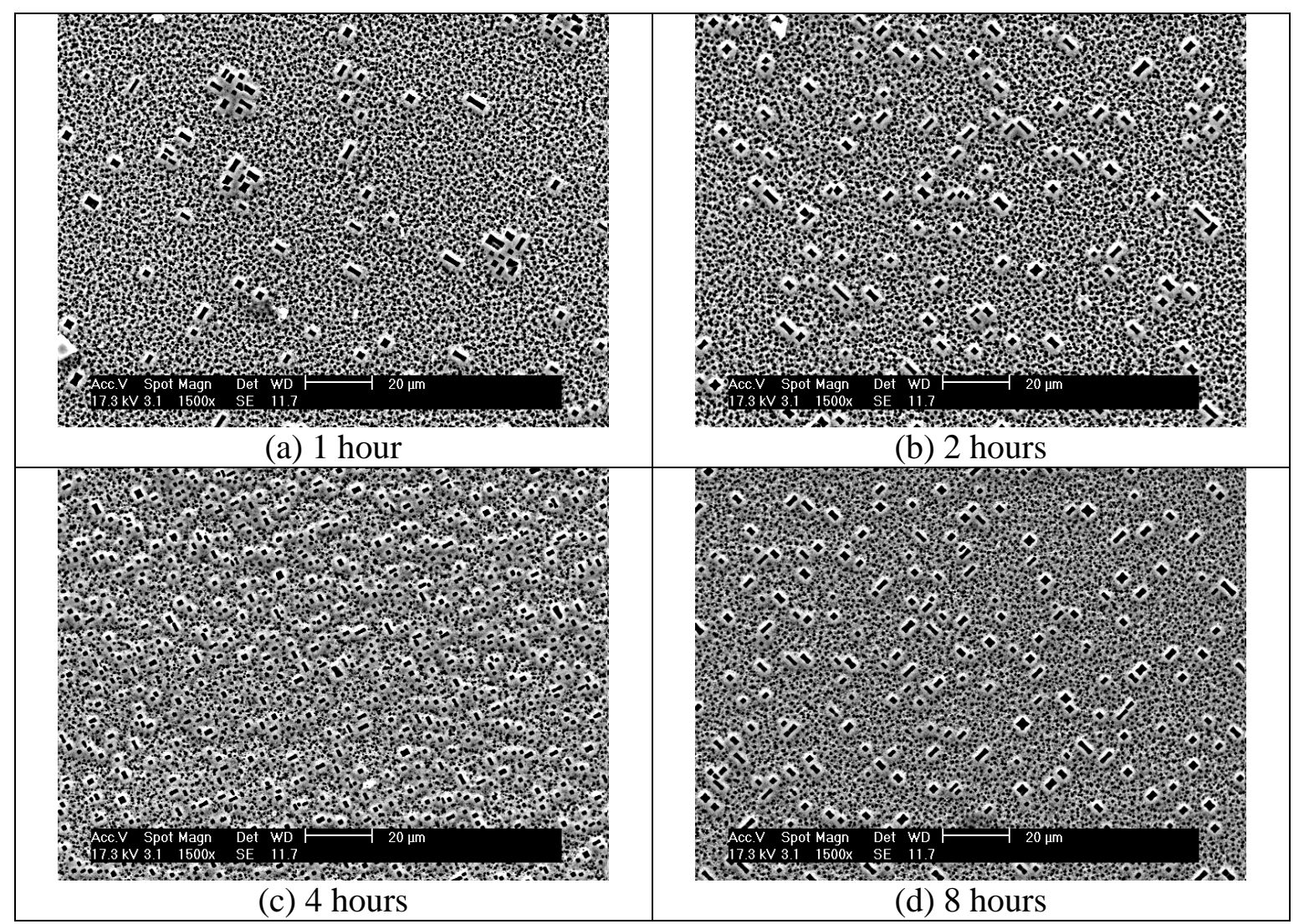

Figure 4. The SEM images of the macropores samples obtained after thermal annealing for (a) 1 hour, (b) 2 hours, (c) 4 hours and (d) 8 hours.

\section{Conclusions}

The new correlation-based granulometry showed to be an effective tool for SEM images processing, in order to analyze quantitavely the porous materials parameters such as pores radii, the size distribution and pores density. Additionally, the proposed granulometry allowed us to classify the pores as circular or square ones. The developed algorithm can contribute in the porous material research area, allowing rapid analysis of structures and rapid extraction of their geometric parameters. The proposed algorithm showed to be an excellent tool for discussing the square pores formation mechanism in silicon substrate by electrochemical anodization process. The results obtained point out that the annealing time of the Al metallized silicon wafer before anodization process is an important parameter for controlling the porous density and their sizes.

\section{Acknowledgements}

The authors thank to CNPq for the financial support. 


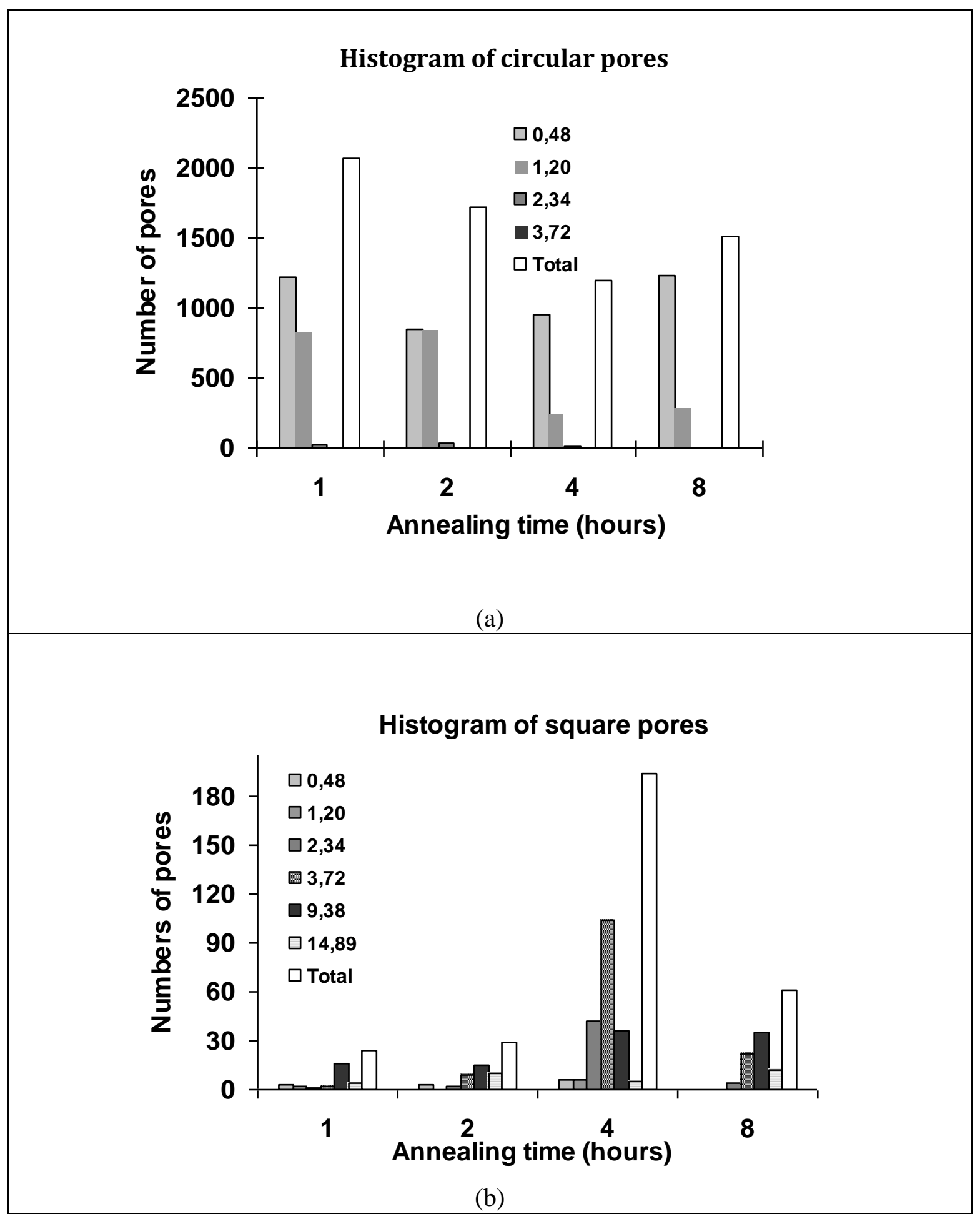

Figure 5. The histograms of circular (a) and square (b) pores obtained from the SEM images. The inner labels describe the pores' areas (in $\mu \mathrm{m}^{2}$ ) corresponding to each bar of the histograms. The white bars represent the total numbers of circular or square pores. 


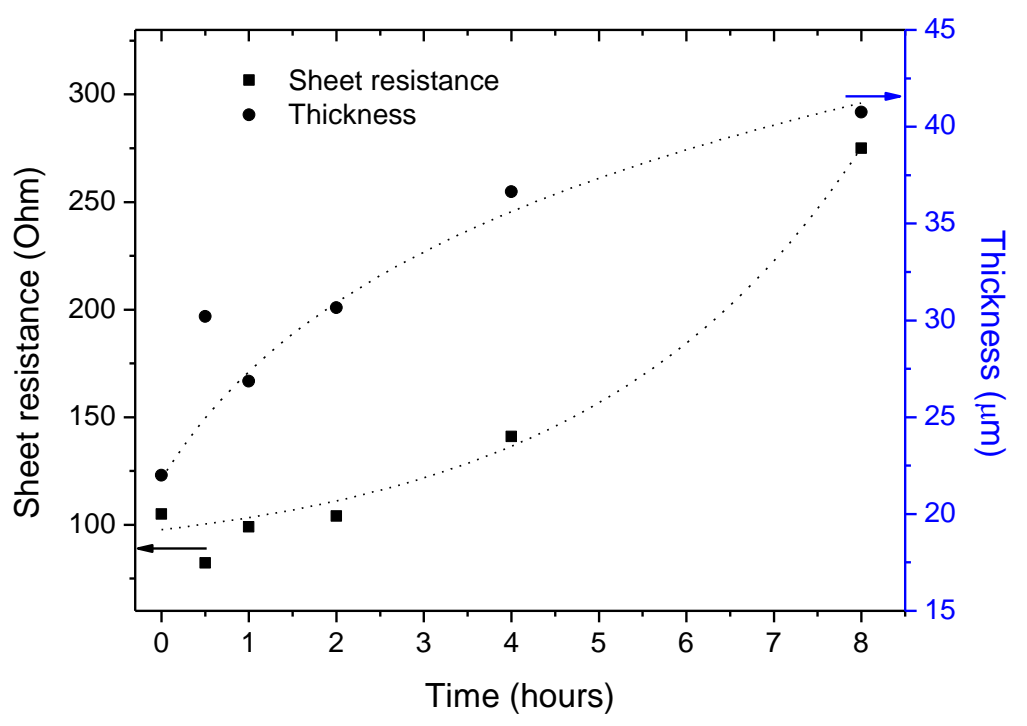

Figure 6. The sheet resistance of the silicon wafer surface as a function of annealing time. The graph depicts also the thickness of porous silicon layers after anodization process.

\section{References}

1. E. R. Dougherty, J. T. Newell and J. B. Pelz, "Morphological Texture-Based Maximum-Likelihood Pixel Classification Based on Local Granulometric Moments," Pattern Recognition, vol. 25, no. 10, pp. 1181-1198 (1992).

2. D. S. Raimundo, P. B. Calipe; D. R. Huanca; W. J. Salcedo, "Anodic Porous Alumina Structural Characteristics Study Based on SEM Image Processing and Analysis," Microelectronics Journal, v. 40, p. 844-847 (2009).

3. Luc Vincent, "Fast Grayscale Granulometry Algorithms," Proc. Int. Symp. Mathematical Morphology, Fontainebleau (1994).

4. Mathworks Image Processing Toolbox 6.4 demo "Granulometry of Snowflakes", accessed on Dec 23, 2009.

http://www.mathworks.com/products/image/demos.html?file=/products/demos/shipping/images/ipexsnow.html

5. N. H. Maerz, T. C. Palangio and J. A. Franklin, "WipFrag image based granulometry system," Proc. of the FRAGBLAST, 5 Workshop on Measurement of Blast Fragmentation, Montreal, pp. 91-99, 23-24 Aug., 1996.

6. H. Föll, M. Christophersen, J. Carstensen and G. Hasse, "Formation and Application of Porous Silicon," Materials Science and Engineering: R: Reports, vol. 39, no. 4, pp. 93-141 (2002). 